$1-11-2022$

\title{
Does the Type of Records Affect the Estimates of the Parameters?
}

Ayush Tripathi

Banaras Hindu University, tripathiayush0@gmail.com

Umesh Singh

Banaras Hindu University

Sanjay Kumar Singh

Banaras Hindu University

Follow this and additional works at: https://digitalcommons.wayne.edu/jmasm

Part of the Applied Statistics Commons, Social and Behavioral Sciences Commons, and the Statistical Theory Commons

\section{Recommended Citation}

Tripathi, A., Singh, U., \& Singh, S. K. (2020). Does the Type of Records Affect the Estimates of the Parameters? Journal of Modern Applied Statistical Methods, 19(1), eP2863. https://doi.org/10.22237/ jmasm $/ 1608552300$

This Regular Article is brought to you for free and open access by the Open Access Journals at DigitalCommons@WayneState. It has been accepted for inclusion in Journal of Modern Applied Statistical Methods by an authorized editor of DigitalCommons@WayneState. 


\section{Does the Type of Records Affect the Estimates of the Parameters?}

\author{
Ayush Tripathi \\ Banaras Hindu Univ. \\ Varanasi, India
}

\author{
Umesh Singh \\ Banaras Hindu Univ. \\ Varanasi, India
}

\author{
Sanjay Kumar Singh \\ Banaras Hindu Univ. \\ Varanasi, India
}

The maximum likelihood estimation of the unknown parameters of inverse Rayleigh and exponential distributions are discussed based on lower and upper records. The aim is to study the effect of the type of records on the behavior of the corresponding estimators. Mean squared errors are calculated through simulation to study the behavior of the estimators. The results shall be of interest to those situations where the data can be obtained in the form of either of the two types of records and the experimenter must decide between these two for estimation of the unknown parameters of the distribution.

Keywords: Record values, Inverse Rayleigh (IR) Distribution, Exponential Distribution, Maximum Likelihood (ML) Estimation, Mean Squared Error (MSE)

\section{Introduction}

In many real-life applications, record value theory and associated statistics are of great importance such as in the fields of meteorology, hydrology, climatology, sports, stock market analysis. For example, meteorologists may be interested in the lowest and highest temperature records and precipitation levels. There are many real-life situations in which we only have record values in our hand, for example in sports events only record-breaking performances are available. The study of records in the statistical sense is, thus, of vital importance. The study of record values became apparent in a climacteric paper by Chandler (1952). In fact, Chandler is the originator of the record value theory and has provided basic properties of the record values. Since then, a number of authors have worked on record values and problems related to it. For more details on record values reader may refer to Foster and Stuart (1954), Galambos (1978), Arnold et al. (1992), Ahsanullah (1995) and Kamps (1995). Sometimes record values can give better

https://doi.org/10.22237/jmasm/1608552300 | Accepted: Apr 25, 2018; Published: Jan 11, 2022.

Correspondence: Ayush Tripathi, tripathiayush0@gmail.com 


\section{TRIPATHI ET AL.}

insight into the unknown phenomenon. The Fisher information contained in a random sample and that contained in a set of record values (lower or upper) has been compared by Ahmadi and Arghami (2001) and it was shown that for some distributions the information contained in a set of first $n$ record values is greater than that contained in a set of $n$ independently identically distributed observations. Here, consider the problem of parameter estimation of the lifetime distributions based on the observed record values.

Although the exponential distribution is most widely discussed distribution in life testing and reliability studies, Inverse Rayleigh (IR) distribution is one of the lifetime distributions which has gained importance in survival analysis in recent years. The IR distribution was introduced by Trayer (1964). Voda (1972) studied some properties of ML estimator for IR distribution based on a random sample and commented that the lifetime distribution of several types of experimental units can be approximated by the IR distribution. Five measures of location for the IR distribution have been suggested by Gharraph (1993). Rao et al. (2013) considered the problem of estimating the stress-strength reliability for the IR distribution. For the study of stress-strength reliability, the reader is referred to Kotz and Pensky (2003). Bayesian prediction bounds for the $s^{\text {th }}$ future record value have been obtained by Al-Hussaini and Ahmad (2003). El-Helbawy (2005) has considered the Bayesian estimation of the parameters along with one and two sample predictions problems for the IR distribution under various loss functions. Dey (2012) has obtained Bayes estimator of the unknown parameter and reliability function and also constructed the HPD intervals for the parameter and reliability function. Bayesian and non-Bayesian estimations of the parameters of the IR distribution and the Bayesian prediction based on lower record values has been attempted by Soliman et al. (2010). Dey (2012) has presented the Bayes estimators of an IR distribution under squared error and linear exponential loss functions.

In this study, the second distribution considered is the exponential distribution, keeping in view it's wide applicability in reliability theory. Some characterization results of the exponential distribution based on the records have already been obtained by Ahsanullah (1978). Dunsmore (1983) has given Bayesian prediction bounds for a future record from the exponential distribution. Samaniego and Whitaker (1986) have considered the likelihood estimation and discussed the conditional unbiased estimator of the mean of the exponential distribution. Arnold et al. (1998) have obtained the ML estimates and best linear unbiased estimator (BLUE) for the exponential distribution. Bayes and empirical Bayes estimators for the unknown parameters based on record values of the generalized exponential distribution have been derived by Jaheen (2004). A Bayes estimation and prediction 


\section{DO TYPE OF RECORDS AFFECT ESTIMATES OF PARAMETERS?}

procedure for two-parameter exponential distribution under linear exponential loss function (LINEX) has been discussed by Ahmadi et al. (2005). Doostparast and Balakrishnan (2009) have discussed the sample size determination for record data and associated cost analysis for the exponential distribution.

Most of the works mentioned above provide the method of the estimation of the parameter of the distribution, although a few presents the method of estimation based on record values, authors considered either the lower or upper record values are available to them for estimation purposes. Some works on parameter estimation based on record values were Nadar \& Kizılaslan (2014), Kiz1laslan \& Nadar (2015), and Singh et al. (2016). A natural query that may arise is, "If it is possible to get both upper as well as lower records on a characteristic assumed to follow a specified family of distribution, does the performance of the estimators of the parameters depend on the type of the records used?"

For example, suppose the water level of a river at a particular place follows some distribution and unknown parameters need to be estimated. The data can be made available in the form of lower records or upper records. Thus, the question arises which type of record should be use for the estimation of parameters. No attempt was made to compare the performances of the estimators based on upper and lower records for the assumed distribution. Thus, the present paper aims to provide an insight into this direction by considering the problem of point estimation of parameters of the above mentioned two models. The aim of this article is to study the properties of maximum likelihood (ML) estimators of the parameters of IR and exponential models based on lower as well as upper records and then study the effect of the type of record on the efficiency of the corresponding estimator. For this purpose, ML estimates and corresponding mean squared error were obtained through the simulation study for parameters of both the distributions based on upper as well as lower records

\section{Maximum Likelihood Estimation Based on Record Values}

Let $X_{1}, X_{2}, X_{3}, \ldots$ be a sequence of independent observations on a random variable $X$ having the cumulative distribution function (cdf) $F(x)$ and probability density function (pdf) $f(x)$. An observation $X_{j}$ is called a record value (or simply a record) if its value is smaller than or greater than all the preceding observations. If it is smaller than all the preceding observations, it is called lower record. Hence $X_{j}$ is a record, if $X_{j}<X_{i}$ for every $i<j$. An analogous definition can be given for upper record also. It is interesting to note here that the first observation is always a lower record as well as upper record. Let $r_{l(1)}, r_{l(2)}, r_{l(3)}, \ldots, r_{l(m)}$ be $m$ lower records, and 


\section{TRIPATHI ET AL.}

then the likelihood function based on $m$ lower records is given by (see Arnold et al., 1998).

$$
L(a ; \underline{r})=f\left(r_{l(m)}\right) \prod_{i=1}^{m-1} \frac{f\left(r_{l(i)}\right)}{F\left(r_{l(i)}\right)}
$$

Similarly, suppose $R_{u(1)}, R_{u(2)}, \ldots, R_{u(n)}$ be the set of $n$ upper records, likelihood function based on $n$ upper records, is given by (see Arnold et al., 1998).

$$
L(a ; \underline{R})=f\left(R_{u(n)}\right) \prod_{i=1}^{n-1} \frac{f\left(R_{u(i)}\right)}{1-F\left(R_{u(i)}\right)}
$$

\section{Estimation for Inverse Rayleigh Distribution}

The probability density function (pdf) and cumulative distribution function (cdf) of the IR distribution with scale parameter $\alpha$ are given below

$$
\begin{gathered}
f(x)=\frac{2 \alpha}{x^{3}} \exp \left(-\frac{\alpha}{x^{2}}\right) \quad x, \alpha>0 \\
F(x)=\exp \left(-\frac{\alpha}{x^{2}}\right) \quad x, \alpha>0
\end{gathered}
$$

\section{Case (a): Maximum Likelihood Estimation Based on Lower Record Values}

Suppose $r_{l(1)}, r_{l(2)}, r_{l(3)}, \ldots, r_{l(m)}$ be the $m$ lower records, arising from the IR distribution with probability density function (3) and corresponding distribution function (4). Likelihood function can be easily obtained from equations (1), (3) and (4) and written as

$$
L(\alpha ; \underline{r})=\left(\frac{2 \alpha}{r_{l(m)}^{3}}\right) \exp \left(-\frac{\alpha}{r_{l(m)}^{2}}\right) \prod_{i=1}^{m-1}\left(\frac{2 \alpha}{r_{l(i)}^{3}}\right)
$$




\section{DO TYPE OF RECORDS AFFECT ESTIMATES OF PARAMETERS?}

After taking the logarithm of the likelihood function, we get

$$
\ln (L(\alpha ; \underline{r}))=\ln (2 \alpha)-3 \ln \left(r_{l(m)}\right)-\left(\frac{\alpha}{r_{l(m)}^{2}}\right)+\sum_{i=1}^{m-1}\left(\ln \left(\frac{2 \alpha}{r_{l(i)}^{3}}\right)\right)
$$

Differentiate the above equation with respect to the parameter $\alpha$ and put it equal to zero to get the likelihood equation, which can be easily solved for $\alpha$. The ML estimate of the parameter $\alpha$ obtained in this way is given below:

$$
\widehat{\alpha_{1}}=(m) r_{l(m)}^{2}
$$

\section{Case (b): Maximum Likelihood Estimation Based on Upper Record Values}

Let $R_{u(1)}, R_{u(2)}, \ldots, R_{u(n)}$ be the set of upper records of size $n$ from IR distribution. Likelihood function can be obtained by using equations (2), (3) and (4) as follows:

$$
L(\alpha ; \underline{R})=\left(\frac{2 \alpha}{R_{u(n)}^{3}}\right) \exp \left(-\frac{\alpha}{R_{u(n)}^{2}}\right) \prod_{i=1}^{n-1} \frac{\left(\frac{2 \alpha}{R_{u(i)}^{3}}\right) \exp \left(-\frac{\alpha}{R_{u(i)}^{2}}\right)}{1-\exp \left(-\alpha / R_{u(i)}^{2}\right)}
$$

After taking the natural logarithm of the likelihood function we get the loglikelihood as

$$
\ln (L(\alpha ; \underline{R}))=(n) \ln (2 \alpha)-3 \sum_{i=1}^{n} \ln \left(R_{u(i)}\right)-\sum_{i=1}^{n}\left(\frac{\alpha}{R_{u(i)}^{2}}\right)-\sum_{i=1}^{n-1} \ln \left(1-\exp \left(-\left(\frac{\alpha}{R_{u(i)}^{2}}\right)\right)\right)
$$

Differentiating above equation with respect $\alpha$ to and equating it to the zero, we get the likelihood equation for $\alpha$ as 


$$
\frac{n}{\alpha}-\sum_{i=1}^{n}\left(\frac{1}{R_{u(i)}^{2}}\right)-\sum_{i=1}^{n-1} \frac{\exp \left(-\frac{\alpha}{R_{u(i)}^{2}}\right)}{1-\exp \left(-\frac{\alpha}{R_{u(i)}^{2}}\right) R_{u(i)}^{2}}=0
$$

By solving above non-linear equation, obtain the ML estimate of parameter, $\widehat{\alpha}_{u}$, based on $n$ upper record values. It is evident a closed form solution of the above equation does not exist. However, numerical methods can be used to get the solution. For IR distribution, ML estimate for $\alpha$ has been discussed previously by Soliman et al. (2010) based on lower record whereas the ML estimate of the parameter based on upper record has not been obtained. For comparison purpose, we have obtained ML estimates for $\alpha$ using lower as well as upper records. It is interesting to note here that the estimator based on the lower records is in nice closed form but the estimator based on the upper records is a solution of a nonlinear equation and thus exact expression for it does not exist.

\section{Estimation for Exponential Distribution}

The pdf and cdf of the one-parameter exponential distribution are given, respectively, by

$$
\begin{gathered}
f(x)=\left(\frac{1}{\sigma}\right) e^{-\frac{x}{\sigma}} \quad x \geq 0, \sigma>0 \\
F(x)=1-e^{-\frac{x}{\sigma}} \quad x \geq 0, \sigma>0
\end{gathered}
$$

\section{Case (a): Maximum Likelihood Estimation Based on Lower Record Values}

Let $r_{l(1)}, r_{l(2)}, r_{l(3)}, \ldots, r_{l(m)}$ be the $m$ lower records from the exponential distribution with pdf (9) and cdf (10). The likelihood function based on $m$ record values, using equations (1), (9) and (10) is given by 
DO TYPE OF RECORDS AFFECT ESTIMATES OF PARAMETERS?

$$
L(\sigma, \underline{r})=\left(\frac{1}{\sigma}\right) \exp \left(-\frac{r_{(m)}}{\sigma}\right) \prod_{i=1}^{m-1} \frac{\left(\frac{1}{\sigma}\right) e^{-\frac{r_{(i)}}{\sigma}}}{1-e^{-\frac{r_{(i)}}{\sigma}}}
$$

The natural logarithm of the likelihood function equation (11) is

$$
\ln (L(\sigma, \underline{r}))=-\ln (\sigma)-\frac{r_{l(m)}}{\sigma}+\sum_{i=1}^{m-1} \ln \left(\frac{\left(\left(\frac{1}{\sigma}\right) e^{-\frac{r_{(i)}}{\sigma}}\right)}{\left(1-e^{-\frac{r_{(i())}}{\sigma}}\right)}\right)
$$

On differentiating above equation with respect to the parameter $\sigma$ and equating to zero, we get

$$
-\frac{m}{\sigma}+\frac{1}{\sigma^{2}} \sum_{i=1}^{m} r_{l(i)}+\sum_{i=1}^{m-1}\left(\frac{\left(r_{l(i)}\right) e^{-\frac{r_{(i)}}{\sigma}}}{\left(1-e^{-\frac{r_{(i)}}{\sigma}}\right) \sigma^{2}}\right)=0
$$

Obtain the ML estimate of parameter, say $\widehat{\sigma}_{l}$, by solving the above non-linear equation. It may be noted here that equation (12) is a non-linear equation and a closed form solution does not exist. However, numerical solution can easily be obtained.

\section{Case (b): Maximum Likelihood Estimation Based on Upper Record Values}

Suppose $R_{u(1)}, R_{u(2)}, \ldots, R_{u(n)}$ are $n$ upper records from the exponential distribution. Likelihood function based on $n$ upper record values can be obtained from (2), (9) and (10) and is given by 


$$
L(\sigma, \underline{R})=\frac{1}{\sigma} \exp \left(-\frac{R_{u(n)}}{\sigma}\right) \prod_{i=1}^{n-1} \frac{1}{\sigma}
$$

Log-likelihood function is given by

$$
\ln (L(\sigma, \underline{R}))=-\ln (\sigma)-\frac{R_{u(n)}}{\sigma}-\sum_{i=1}^{n-1} \ln (\sigma)
$$

Differentiate above equation with respect to the parameter $\sigma$ and equating to zero, we get the ML estimate of $\sigma$ based on $n$ upper record as

$$
\widehat{\sigma_{u}}=\frac{R_{u(n)}}{n}
$$

The above case (b) has been considered previously by Arnold et al. (1998). However, no attempts have been made to discuss ML estimate of the parameter based on lower records for the exponential distribution (discussed in case (a)). It is worthwhile to mention here that for IR distribution the maximum likelihood estimator of the parameter based on lower records is in nice closed form but it depends only on the lowest lower record whereas the maximum likelihood estimator of the parameter based on upper records is not obtainable in nice closed form but it utilizes all the upper records. Contrary to it, the maximum likelihood estimator of the parameter of the exponential distribution based on lower records is not in nice closed form but utilizes all the lower records whereas the estimator based on upper record is in closed form although it utilizes only the highest upper record. As mentioned earlier, this motivated us to check whether the performance of the maximum likelihood estimator depends on the type of records used and further if there exists any such dependency, does it vary from distribution to distribution. For the study of the performance of the estimator, consider the use of mean square error criterion. The exact expression for the mean square error of the estimators is not obtainable in a closed form particularly in those situations where the estimator does not have the closed form. Therefore it is proposed to carry out a simulation study given in the next section. 


\section{DO TYPE OF RECORDS AFFECT ESTIMATES OF PARAMETERS?}

\section{Simulation}

A simulation study is conducted to compare the performance of ML estimators based on lower and upper record values. Samples of lower and upper records are generated from the considered models for arbitrarily chosen values of the parameter. The comparison of the performance of the estimators has been made on the basis of mean squared error (MSE). For this purpose, we first generated random samples of large size called here as the complete sample from the specified distribution. Then the desired numbers of record values (i.e. the sample of record values) were obtained. Based on the records, thus obtained, the estimate of the parameter is computed. The process is repeated sufficiently large number of times to get the estimated MSE of the estimator. In order to study the effect of the number of records on the MSE of the estimator, we varied the number of records from the generated complete sample.

For IR model, let $\alpha=0.1$ (, 0.5, 1 and 1.2). The various values of the number of records considered for the estimation of the parameter are 3, 5, 7 and 9 for both lower as well as upper records. The samples of record values were simulated using the procedure mentioned above and then the estimate of the parameter is calculated. These steps are repeated $N=30,000$ times to get the estimated mean squared error (MSE) of the ML estimate of the parameter $(\alpha)$. The results obtained in this way are summarized and presented in Table 1. A similar process has been followed for exponential distribution also by taking the value of the parameter arbitrarily as $\sigma=0.1$ (, 0.5, 1 and 1.2); keeping the number of records (record sample size) and the number of the repetition of sampling process same as that for IR model. The MSEs of the ML estimators of the parameters based on lower as well as upper records are presented in Table 2. The average estimate of the parameter is provided along with corresponding MSE and bias on the complete sample from which record values have been obtained. The average estimate in each case is close to the true value with very small MSE and Bias. The sample size in each case is 9,000 which is very large.

From Table 1, as the record sample size increases the MSE as well as the bias of the estimator based on upper as well as lower record values decreases. The ML estimate of the unknown parameter of IR distribution based on lower record values has the smaller MSE and bias as compared to that based on upper record values. The estimate based on upper as well lower records, both have a positive bias. 


\section{TRIPATHI ET AL.}

Table 1. Estimate of the parameter $(\alpha)$ of IR distribution along with corresponding MSE and bias

\begin{tabular}{|c|c|c|c|c|c|c|c|c|}
\hline \multirow{3}{*}{$\begin{array}{l}\text { Value of } \\
\text { Parameter }\end{array}$} & \multirow{3}{*}{$\begin{array}{c}\text { Complete } \\
\text { Sample } \\
\text { Estimate } \\
\text { (MSE) } \\
\text { (Bias) }\end{array}$} & \multirow{3}{*}{ \# Records } & \multicolumn{3}{|c|}{ Lower } & \multicolumn{3}{|c|}{ Upper } \\
\hline & & & $\begin{array}{l}\text { Average } \\
\text { Estimate }\end{array}$ & MSE & Bias & $\begin{array}{c}\text { Average } \\
\text { Estimate }\end{array}$ & MSE & Bias \\
\hline & & & & & & & & \\
\hline \multirow{4}{*}{$\alpha=0.1$} & \multirow{4}{*}{$\begin{array}{c}0.100008 \\
(0.000001) \\
(0.000008)\end{array}$} & 3 & 0.1912 & 0.0359 & 0.0912 & 0.2064 & 0.1457 & 0.1064 \\
\hline & & 5 & 0.1590 & 0.0094 & 0.0590 & 0.2008 & 0.1277 & 0.1008 \\
\hline & & 7 & 0.1482 & 0.0050 & 0.0482 & 0.1993 & 0.1220 & 0.0993 \\
\hline & & 9 & 0.1414 & 0.0033 & 0.0414 & 0.1990 & 0.1207 & 0.0990 \\
\hline \multirow{4}{*}{$\alpha=0.5$} & \multirow{4}{*}{$\begin{array}{c}0.500014 \\
(0.000028) \\
(0.000014)\end{array}$} & 3 & 0.9550 & 0.9179 & 0.4550 & 1.0415 & 4.5579 & 0.5415 \\
\hline & & 5 & 0.7983 & 0.2528 & 0.2983 & 1.0122 & 3.8905 & 0.5122 \\
\hline & & 7 & 0.7444 & 0.1295 & 0.2444 & 1.0042 & 3.6743 & 0.5043 \\
\hline & & 9 & 0.7087 & 0.0841 & 0.2087 & 1.0028 & 3.6187 & 0.5028 \\
\hline \multirow{4}{*}{$\alpha=1.0$} & \multirow{4}{*}{$\begin{array}{c}1.000105 \\
(0.000109) \\
(0.000105)\end{array}$} & 3 & 1.9418 & 4.6779 & 0.9418 & 2.0753 & 15.5592 & 1.0753 \\
\hline & & 5 & 1.5994 & 1.0096 & 0.5994 & 2.0177 & 13.7187 & 1.0177 \\
\hline & & 7 & 1.4874 & 0.5250 & 0.4874 & 2.0029 & 13.1315 & 1.0029 \\
\hline & & 9 & 1.4189 & 0.3428 & 0.4189 & 1.9996 & 12.9613 & 0.9996 \\
\hline \multirow{4}{*}{$\alpha=1.2$} & \multirow{4}{*}{$\begin{array}{c}1.200169 \\
(0.000159) \\
(0.000169)\end{array}$} & 3 & 2.2914 & 5.9044 & 1.0914 & 2.5005 & 22.2708 & 1.3005 \\
\hline & & 5 & 1.9060 & 1.3664 & 0.7060 & 2.4326 & 19.3794 & 1.2326 \\
\hline & & 7 & 1.7778 & 0.7328 & 0.5778 & 2.4150 & 18.5095 & 1.2150 \\
\hline & & 9 & 1.6961 & 0.4736 & 0.4961 & 2.4118 & 18.3262 & 1.2118 \\
\hline
\end{tabular}

Table 2 contains the entries for exponential distribution similar to those contained in Table 1 for IR distribution. The ML estimate of the parameter of exponential distribution based on upper record values has smaller MSE than that based on lower record values. The bias in the estimates based on lower record is positive whereas it is negative for the estimates based on upper record.

The magnitude of the bias decreases as the number of records used for the estimation increases in both the cases. However, the magnitude of the bias of the estimate based on lower record is often (except for estimates based on 3 records) less than those based on upper record. As the number of records increases, MSE and bias decreases. 


\section{DO TYPE OF RECORDS AFFECT ESTIMATES OF PARAMETERS?}

Table 2. Estimate of the parameter $(\sigma)$ of exponential distribution along with corresponding MSE and bias

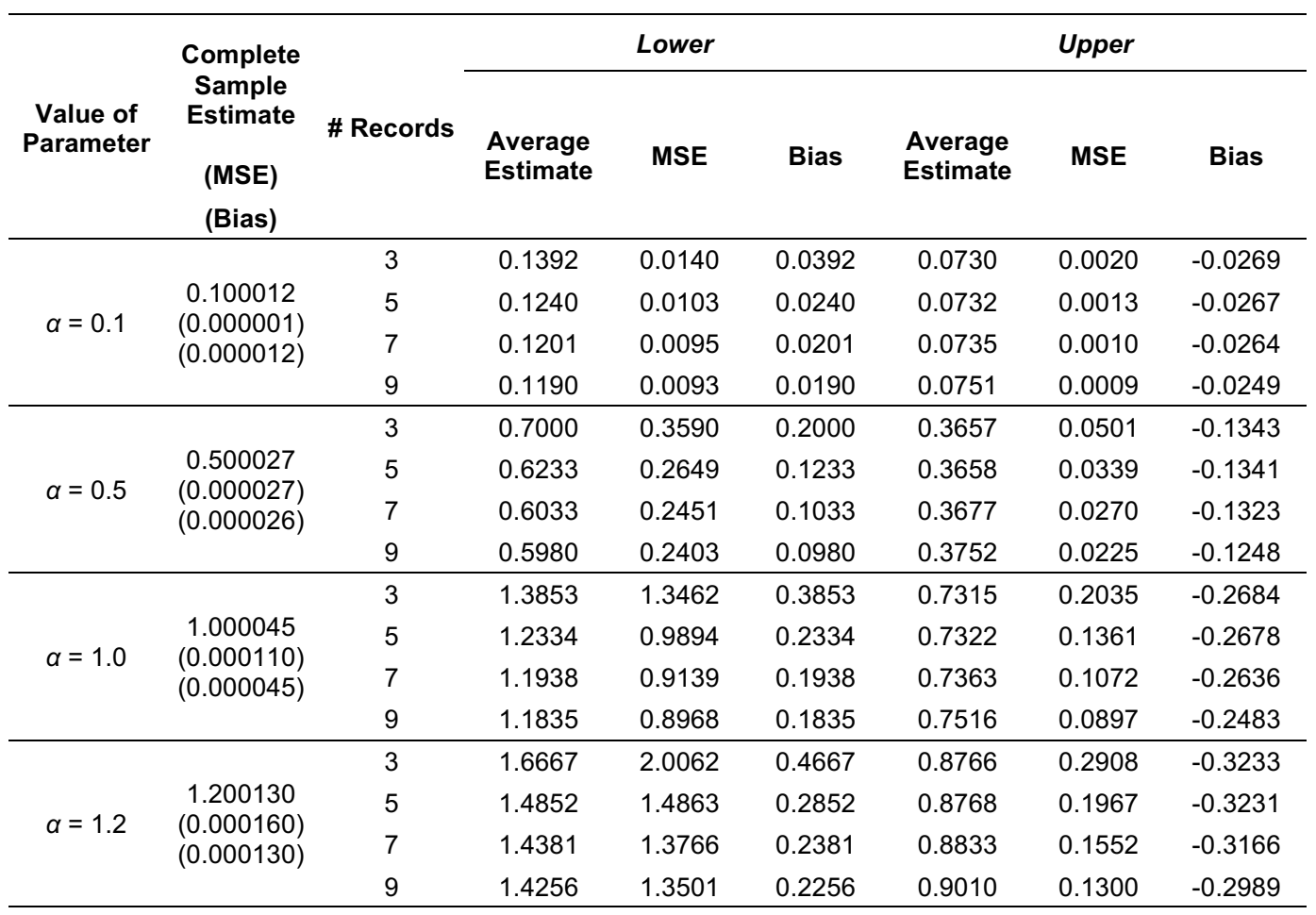

\section{Conclusion}

The aim of this article is to consider the effect of type of records on the estimates of the parameter of the distribution from which the records arise based on the IR distribution and exponential distribution. For estimation of the parameter of IR distribution, the lower records provide a better estimate than that based on the upper record in the sense of having smaller MSE and less bias. But the contrary is the situation for the estimation of the parameter of the exponential distribution. In this case, the estimates based on upper records are better than that based on the lower record because of having smaller MSE, although the absolute bias is slightly higher. The performance of the estimator depends on the type of records. However, suitability of the type of record varies from distribution to distribution. The estimates based on the records will be less precise than that based on the complete sample which gives rise to the record values. 


\section{TRIPATHI ET AL.}

\section{References}

Ahmadi, J., Doostparast, M., \& Parsian, A. (2005). Estimation and prediction in a two-parameter exponential distribution based on k-record values under linex loss function. Communications in Statistics-Theory and Methods, 34(4), 795-805. https://doi.org/10.1081/sta-200054393

Ahmadi, J., \& Arghami, N. R. (2001). On the Fisher information in record values. Metrika, 53(3), 195-206. https://doi.org/10.1007/s001840000089

Ahsanullah, M. (1978). Record values and the exponential distribution. Annals of the Institute of Statistical Mathematics, 30(3), 429-433. https://doi.org/10.1007/bf02480233

Ahsanullah, M. (1995). Record statistics. New York: Nova Science Publishers.

Al-Hussaini, E. K., \& Ahmad, A. E. B. A. (2003). On Bayesian interval prediction of future records. Test, 12(1), 79-99. https://doi.org/10.1007/bf02595812

Arnold, B. C., Balakrishnan, N., \& Nagaraja, H. N. (1998). Records. New York: John Wiley \& Sons. https://doi.org/10.1002/9781118150412

Arnold, B. C., Balakrishnan, N., \& Nagaraja, H. N. (1992). A first course in order statistics. New York: SIAM, John Wiley \& Sons. https://doi.org/10.1137/1.9780898719062

Chandler, K. N. (1952). The distribution and frequency of record values. Journal of the Royal Statistical Society. Series B (Methodological), 14(2), 220-228. https://doi.org/10.1111/j.2517-6161.1952.tb00115.x

Dey, S. (2012). Bayesian estimation of the parameter and reliability function of an inverse Rayleigh distribution. Malaysian Journal of Mathematical Sciences, 6(1), 113124.

Doostparast, M., \& Balakrishnan, N. (2009). Optimal sample size for record data and associated cost analysis for exponential distribution. Journal of Statistical Computation and Simulation, 80(12), 1389-1401. https://doi.org/10.1080/00949650903150171

Dunsmore, I. R. (1983). The future occurrence of records. Annals of the Institute of Statistical Mathematics, 35(2), 267-277. https://doi.org/10.1007/bf02480982

El-Helbawy, A. A., \& Abd-El-Monem. (2005). Bayesian Estimation and Prediction for the Inverse Rayleigh Lifetime Distribution. Proceeding of the 40st annual conference of statistics, computer sciences and operation research, ISSR, Cairo University, 45-59. 


\section{DO TYPE OF RECORDS AFFECT ESTIMATES OF PARAMETERS?}

Foster, F. G., \& Stuart, A. (1954). Distribution-free tests in time-series based on the breaking of records. Journal of the Royal Statistical Society. Series B (Methodological) 16(1), 1-13. https://doi.org/10.1111/j.2517-6161.1954.tb00143.x

Galambos, J. (1978). The asymptotic theory of extreme order statistics. New York: John Wiley \& Sons.

Gharraph, M. K. (1993). Comparison of estimators of location measures of an inverse Rayleigh distribution. The Egyptian Statistical Journal, 37, 295-309.

Jaheen, Z. F. (2004). Empirical Bayes inference for generalized exponential distribution based on records. Communications in Statistics-Theory and Methods, 33(8), 1851-1861. https://doi.org/10.1081/sta-120037445

Kamps, U. (1995). Recurrence relations for moments of record values. Journal of Statistical Planning and Inference, 45(1-2), 225-234. https://doi.org/10.1016/03783758(94)00073-5

Kizilaslan, F., \& Nadar, M. (2016). Estimation and prediction of the Kumaraswamy distribution based on record values and inter-record times. Journal of Statistical Computation and Simulation, 86(12), 2471-2493. https://doi.org/10.1080/00949655.2015.1119832

Kotz, S., \& Pensky, M. (2003). The stress-strength model and its generalizations: Theory and applications. Singapore: World Scientific Publishers. https://doi.org/10.1142/9789812564511

Nadar, M., \& Kizılaslan, F. (2014). Estimation and prediction of the Burr type XII distribution based on record values and inter-record times. Journal of Statistical Computation and Simulation, 85(16), 3297-3321.

https://doi.org/10.1080/00949655.2014.970554

Rao, G. S., Kantam, R. R. L., Rosaiah, K., \& Reddy, J. P. (2013). Estimation of stress-strength reliability from inverse Rayleigh distribution. Journal of Industrial and Production Engineering, 30(4), 256-263. https://doi.org/10.1080/21681015.2013.828787

Samaniego, F. J., \& Whitaker, L. R. (1986). On estimating population characteristics from record-breaking observations. i. parametric results. Naval Research Logistics (NRL), 33(3), 531-543. https://doi.org/10.1002/nav.3800330317

Singh, S., Tripathi, Y. M., \& Wu, S. J. (2016). Bayesian estimation and prediction based on lognormal record values. Journal of Applied Statistics, 44(5), 916-940. https://doi.org/10.1080/02664763.2016.1189520

Soliman, A., Amin, E. A., \& Abd-El Aziz, A. A. (2010). Estimation and prediction from inverse Rayleigh distribution based on lower record values. Applied Mathematical Sciences, 4(62), 3057-3066. 


\section{TRIPATHI ET AL.}

Trayer, N. (1964). [Response.]. Nauk, Belorus, U.S.S.R.: Doklady Academy.

Voda, V. G. (1972). On the inverse Rayleigh distributed random variable. Rep. Statist. App. Res. (JUSE), 19(4), 13-21. 